\title{
The Free Autografting of Entire Limb Muscles in the Cat: Morphology 1
}

\author{
SHAHZAD A. MUFTI, ${ }^{2}$ BRUCE M. CARLSON, ${ }^{3}$ LEO C. MAXWELL AND \\ JOHN A. FAULKNER \\ Departments of Anatomy and Physiology, University of Michigan, Ann Arbor, \\ Michigan 48109
}

\begin{abstract}
Normal or pre-denervated extensor digitorum longus (EDL) muscles were successfully grafted in place of the contralateral EDL muscles in cats. Histological preparations of 57 grafts were examined at intervals from 4 to 170 days after transplantation. The morphological sequence of events in a cat muscle graft is similar to that in the rat except that the time course of regeneration is considerably slower. Surviving original muscle fibers form a thin rim at the periphery of the graft. Beneath this rim, the original muscle fibers undergo fragmentation, starting near the periphery and progressing toward the center. Regenerating muscle fibers take the place of the degenerated muscle fibers. In pre-denervated grafts, the last of the original muscle fibers in the center of the graft have broken down by $\mathbf{4 1}$ days whereas in normal muscle grafts the original muscle fibers in the central region persist until 50 days. The main difference between grafts of normal and pre-denervated muscles is the rate of breakdown of the original muscle fibers. Long term grafts of both groups are morphologically very similar.
\end{abstract}

The successful free autografting of entire skeletal muscles was first performed on the gastrocnemius muscle in rats by Bosova ('62) and Studitsky and Bosova ('60). Free grafts of the normal gastrocnemius were not successful, but if the muscle was pre-denervated several weeks prior to grafting, the graft was successful. These investigators hypothesized that pre-denervation reduces the muscle to a "plastic condition," which enables the muscle to withstand the unfavorable environmental conditions that occur during the early days after it is grafted. The adaptive changes brought about by pre-denervation are poorly defined.

In 1971, Thompson independently rediscovered the pre-denervation technique and soon performed the first successful free muscle grafts in humans. Since then the free autografting of human skeletal skeletal muscles has come into increasingly widespread use for the clinical treatment of conditions in which there is a functional deficit of a muscle or group of muscles (Thompson, '71a, '74; Hakelius, '74, '75). Progress in further application of this technique is hindered by insufficient knowledge of the factors that account for the success of free muscle grafts.
Major questions at this point are: (1) What do the muscle fibers in a mature free graft represent-surviving original muscle fibers, regenerating muscle fibers, of both? (2) What changes effected by pre-denervation are relevant to the survival and functioning of a muscle graft? (3) What is the most convenient and effective form of pretreatment of a muscle that is to be grafted? and (4) To what extent does a freely grafted muscle approach normal structure and function?

In the present study we have examined the reactions of the extensor digitorum longus (EDL) muscles of the cat after free autografting into the limbs. This report describes the histological changes in these grafts.

\section{MATERIALS AND METHODS}

\section{Operative technique}

In 30 cats, both left and right EDL muscles $(3.3 \pm 0.2 \mathrm{gm})$ were transplanted heterotopically. Each cat received a ketamine preanesthetic $(25 \mathrm{mg} / \mathrm{kg}$, I.M.) and pentobarbital

\footnotetext{
Received May 17, '76. Accepted Mar. 7, '77.

${ }^{1}$ Supported by grants by the Muscular Dystrophy Association to BMC and JAF and an MDA Postdoctoral Fellowship to SAM.

${ }^{2}$ Present address: Department of Zoology, University of the Punjab, New Campus, Lahore, Pakistan.

${ }^{3}$ Send reprint requests to BMC.
} 
anesthetic (10-15 mg/kg I.V.) Anesthesia was maintained by supplementary doses of pentobarbital. Surgery was performed under sterile conditions. A curved incision was made on the lateral side of the lower leg from the origin to the insertion of the EDL muscle. The muscle, wrapped in gauze moistened with warm physiological saline, was elevated and fascial connections were cut or removed by blunt dissection. All vascular connections were ligated and cut, and the muscle was removed and weighed under sterile conditions. The contralateral EDL was removed, and the EDL graft was placed into the bed of the removed muscle. The tendon ends were sutured in place and the fascia and skin were sutured closed. No attempt was made to restore the nervous or vascular connections.

Two types of autotransplants were made. Twenty EDL muscles were transplanted as described, without denervation prior to the actual transplantation. These are termed normal transplants. Forty EDL muscles were transplanted 14 to 28 days after the three nerves to the EDL muscles had been severed. Preliminary experimentation revealed minor differences, at most, in muscles grafted after two to four weeks of pre-denervation. Three weeks was then chosen as the standard predenervation period. The nerves were cut ap. proximately $2 \mathrm{~mm}$ from the point of entry into the muscle. These are termed pre-denervated transplants.

\section{Histological techniques}

Histological preparations were made from 57 grafts in 30 animals. Animals were sacrificed at irregular postoperative intervals $(4$, $8,14,16,26,28,41,49,51,57,70,71,77,90$, $122,133,135,140,153,158,162,180,200$ and 207 days). Representative portions of each graft were fixed in Bouin's solution. From each graft paraffin sections, cut at $7 \mu \mathrm{m}$, were stained in Ehrlich's hematoxylin and eosin and in Heidenhain's aniline blue connective tissue stain (Armed Forces Institute of Pathology, '60). Fifteen $\mu \mathrm{m}$ sections were stained by the Palmgren silver method (Palmgren, '60) for nerve fibers. In addition, frozen sections of grafts taken at 51,71,90, $122,133,135,140,153,158,162,180,200$ and 207 days postoperatively were stained by the Gomori acetylthiocholine method for motor end plates (Pearse, '72).

\section{RESULTS \\ Gross observations}

At the time of grafting the mean weight of 21 normal extensor digitorum longus muscles was $3.84 \pm 0.19$ (S.E.) gm. The mean weight of 39 predenervated muscles was $2.81 \pm 0.14$ gm. At the time of grafting, the mean body weight of all cats was $4.16 \pm 0.16 \mathrm{~kg}$.

Of the 60 EDL muscles transplanted, two normal and two pre-denervated grafts in four different cats were unsuccessful. In the unsuccessful cases, no tissue was present in the bed of the graft. In two early cases ( 8 days) the grafts had liquefied. The fluid was cul. tured and found to be sterile. The cause of the liquefaction remains unknown. This reaction occurred only in the first series of operations.

Four days after transplantation, grafts of normal muscle were larger than normal in diameter and were pale in color. This is due to a temporary edema (Maxwell et al., '77). The tendon sutures were intact, but no tendinous connections were established. There were no tissue or vascular connections to surrounding tissue. By eight days many diffuse small vascular connections were observed between the graft and surrounding tissues, particularly along the proximal third of the grafts. The reestablishment of anatomical continuity between the ends of the graft and the tendon stumps to which they were sutured was noted by 14 days. These tendon connections were still weak, and their gross morphology resembled the fine, fibrous outgrowths seen in the regeneration of tendons in the rat (Carlson, '72). When directly stimulated with a square wave electrical pulse $(0.2 \mathrm{msec}$ duration, stimulus strength up to $8 \mathrm{mv}$ ) applied through stainless steel needle electrodes, the grafts did not contract. The larger masses of the grafts $(4-5 \mathrm{gm})$ persisted up to 16 days, but by 26 days the mean mass of grafts had decreased to less than $0.5 \mathrm{gm}$. Except for their smaller cross-sectional area, pre-denervated grafts differed little from normal grafts in their gross appearance and rate of establishing neurovascular and tendinous connections with the surrounding limb tissues.

Grafts removed between 30 and 170 days after transplantation had many fascial and vascular connections to adjacent tissues. The grafts had regained normal color, and portions of the graft contracted when stimulated directly. Contractions were observed earliest in 
the proximal third of the graft. The mass varied greatly, with a range from $0.92-3.0 \mathrm{gm}$ and a mean of $1.9 \pm 0.5 \mathrm{gm}$. In these later grafts, the tendons appeared normal except for two in which the distal tendons had become attached to the anterior tibial muscle.

\section{Microscopic observations}

\section{Pre-denervated grafts}

Almost the entire cross-sectional area of early grafts was occupied by ischemic muscle fibers, whose fascicular organization remained unchanged from that of a normal muscle (fig. 1). The muscle fibers demonstrated the typical histological features of early ischemic necrosis. The staining reaction of the sarcoplasm was less intense than that of normal muscle fibers, and the fibers took on a hyaline appearance. Scattered discoid degeneration was also present. Pycnosis of subsarcolemmal nuclei was common. Despite these degenerative changes, phagocytic cells were not yet found in the degenerating sarcoplasm and sarcolysis had not yet begun. In the 4-day graft, the periphery of the graft was the site of an acute inflammatory response in which neutrophils were the predominant cells. The inflammatory reaction appeared to penetrate into the graft along the fascial planes of the perimysium and then spread into the endomysial spaces. In the most peripheral areas, there was fragmentation of the sarcoplasm of some muscle fibers, but macrophages were not seen within the sarcoplasm and there was little or no sarcoplasmic removal. At the light microscopic level, there was no evidence that muscle regeneration had begun.

By eight days, the grafts could be subdivided into three zones (1) a thin outer zone containing surviving muscle fibers, (2) a thin intermediate zone containing fragmented original muscle fibers and regenerating new ones and (3) a very large central zone of original muscle fibers that had undergone early degenerative changes, but minimal cytoplasmic removal (fig. 2). Already in the intermediate zone, a radial gradient of maturity of regenerating muscle, ranging from myoblasts near the center to early myotubes toward the periphery, could be seen. The distinction between surviving peripheral and regenerating muscle fibers, on the one hand, and the degenerating muscle fibers of the central zone, on the other, was particularly apparent in sec- tions stained for connective tissue. In addition to lacking any structural abnormalities at the histological level, the viable muscle fibers were red, whereas the necrotic muscle fibers of the central zone were stained a deep purple.

From eight days until early in the third week, there was relatively slow progression inward of the intermediate zone of degeneration and regeneration. Within the intermediate zone the regenerating muscle fibers continued to mature, leading to a more pronounced gradient of maturational changes than was seen in 8-day grafts. At the end of the first month (fig. 3), however, the central zone had shrunk considerably. The zone of re generation was much larger than before, with the spectrum from myoblasts and early myotubes to young muscle fibers being represented in the gradient (fig. 4). The regenerat. ing muscle fibers were already becoming organized into well defined fascicles (fig. 3).

In pre-denervated grafts the central zone of necrotic muscle fibers had disappeared by the end of the sixth week, leaving a small focus of loose connective tissue, phagocytic cells and early stages of muscle fiber regeneration. The remainder of the grafts was occupied prin. cipally by new muscle fibers (fig. 5).

Grafts older than six weeks consisted of varying proportions of muscle fibers and bands of connective tissue (fig. 6). The main developmental activity was the continued maturation of muscle fibers, particularly those toward the centers of the grafts (figs. 7, 8). Older grafts were composed of large masses of muscle fibers, organized into distinct fascicles and surrounded by normal or greater than normal amounts of dense connective tissue (fig. 6).

The presence of nerve fibers among the muscle fibers in the grafts was shown by silver staining. Motor end plates were demonstrated histochemically in a 51-day graft and in later grafts.

\section{Normal grafts}

At eight days the periphery was occupied by a thin basophilic zone containing degenerating old muscle and regenerating new muscle fibers (fig. 9). At the outer border of the central zone, the cytoplasm of old muscle fibers was being removed by invading macrophages in a characteristic pattern that began in the center of the muscle fiber and then expanded toward the sarcolemma (fig. 10). 
From the second through the sixth week, the principal difference between normal and pre-denervated grafts was the relative size of the central zone of necrotic original muscle fibers. In all normal muscle grafts older than eight days the central zones were relatively and absolutely larger than in their pre-denervated counterparts. In our specimens the central zone had disappeared in pre-denervated grafts by day 41 whereas in grafts of normal muscle they lasted longer, up to 49 days. The major difference between grafts of normal and pre-denervated muscle was the length of time required to eliminate the necrotic original muscle fibers from the central zone of the grafts and the consistently larger cross-sectional areas of normal grafts was a reflection of this.

\section{Abnormalities in free muscle grafts}

Several abnormalities in the restorative process were commonly encountered in the grafts. One was the presence of regenerating muscle fibers that were obviously degenerating. Such muscle fibers were seen in varying numbers in virtually all grafts, both normal and predenervated, from 16 days to four months after transplantation. They were always found in the zone of regeneration, either among healthy looking regenerating muscle fibers or in areas with greater than normal amounts of connective tissue. The degenerating muscle fibers were characterized by nuclear pycnosis, and by clumping and flattening of the nuclei perpendicular to the long axis of the muscle fiber. The intensity of sarcoplasmic staining was increased and some sarcoplasmic fragmentation was seen (fig. 11). These changes are similar to those seen in muscle fibers regenerating in denervated limbs (Mong, '75).

Another abnormality was the presence of fascicles or groups of muscle fibers having a much smaller diameter than normal (fig. 7). Although the proportion varied from graft to graft, virtually every graft contained some of these small muscle fibers. No motor end plates were found on the thin muscle fibers. In addition, a heterogeneous staining pattern was not found when these muscle fibers were stained for SDH and ATPase activity (Maxwell et al., '77).

Central nuclei were commonly encountered in cross sections of muscle fibers of mature grafts in cats. Most of the muscle fibers in free grafts of entire muscles were oriented along the normal directions for the muscles in question, but occasionally bundles of muscle fibers with abnormal orientations were present.

\section{DISCUSSION}

There are surprisingly few differences between the histological events occurring in freely grafted EDL muscles of cats and those occurring in free grafts of the EDL muscles in rats (Carlson and Gutmann, '75a). The overall patterns of histological reactions to grafting in both species are similar. The primary differences are the slower rate of these reactions and the lesser degree of synchrony of stages in regenerating cat muscle.

Within two to three days in the rat and one to two weeks in the cat, free muscle grafts can be subdivided into three major zones (Carlson and Gutmann, '75a; Schiaffino et al., '75). The outer zone is a thin peripheral rim that contains a few layers of intact muscle fibers that have obviously survived the early avascular period after grafting and have become revascularized before irreversible damage to them has occurred. The intermediate, or middle, zone is an area of intense cellular activity, involving primarily the degeneration of old and the regeneration of new muscle fibers. The central zone contains original muscle fibers in a state of ischemic necrosis. There are abundant signs of nuclear and cytoplasmic damage, but sarcoplasmic removal has not yet begun.

A recognition of the cellular activities in the intermediate zone of free grafts is the key to understanding the overall success of the free grafting procedures. An early free muscle graft can be divided into two regions. Around the periphery is a thin zone in which sufficient oxygen and substrates are available to preserve the muscle fibers in their original state. The remainder of the graft (internal to the thin peripheral zone) consists of muscle fibers that have been damaged to such an extent that they cannot survive as intact entities. These muscle fibers, isolated from a blood supply, undergo both nuclear and sarcoplasmic degeneration. Sarcoplasmic removal begins when macrophages, apparently provided by the vasculature growing into the graft, enter the muscle fibers and engulf sarcoplasmic fragments. In the devascularized state, there appears to be the selective survival of mononucleated (satellite?) cells situated beneath the basement membrane of the muscle fiber (Snow, '73). 
While cytoplasmic fragmentation is underway, myoblastic cells beneath the basement membrane of the muscle fiber becomes activated, and ultimately myotubes form. In free grafts, the region in which both the degeneration of old and the regeneration of new muscle fibers is taking place is called the intermediate zone (Schiaffino et al., '75). This zone in free grafts of both cat and rat muscle includes the zones that have been called the peripheral and transitional zones in minced muscle regenerates (Carlson, '72). Minced muscle regenerates do not have an outer zone of surviving muscle fibers.

After the intermediate zone of a free graft is initially established, it expands centripetally at the expense of the central zone. This inward expansion appears to be correlated with increasing penetration of new vascular channels into the interior of the graft, but this has not been directly demonstrated in free grafts of any species. The net result of this progressive inward expansion of the intermediate zone is the establishment of a radial gradient of maturity of regenerating muscle fibers, with the most mature ones at the periphery and the least mature along the inner border of this zone (figs. 4, 5). In the cat, as opposed to the rat, the intermediate zone appears to contain an unknown percentage of greatly atrophied original muscle fibers (Schiaffino et al., '75). In our cat preparations, it was not possible to make any quantitative assessments of the presence or number of original muscle fibers surviving in the peripheral and intermediate zones because the temporal and spatial separations between degenerating, regenerating, and surviving muscle fibers are not so clear as they are in the rat (Carlson, '76).

The relative success of free grafts of normal muscle was an unexpected finding in our experiments. It is well established that in the rat there is little difference between long term free grafts of normal and pre-denervated EDL or soleus muscles although there are some distinct differences in both morphology and contractile properties during the first week (Carlson and Gutmann, '75a,b; Vyskočil et al., '73). However, free grafts of large muscles (e.g., the gastrocnemius) in the rat are much less successful if the muscle is not predenervated (Bosova, '62; Zhenevskaya, '68).

In the cat, the most obvious difference between normal and pre-denervated grafts is that normal grafts require a longer time for the disappearance of the central zone of original muscle fibers. Compared with normal muscle, pre-denervated muscles of frogs ( $\mathrm{Hsu}$, '74), mice (Yeasting, '69) and rats (Carlson and Gutmann, '75a) show a pronounced acceleration of the earliest (pre-myotube) stages of regeneration. This is presumably due to the increase in numbers and activation of potentially myogenic cells before the muscle fibers are traumatized by grafting. The origin of these cells remains in question. Ontell ('74) has discussed a number of possible models that would account for changes in the number of satellite cells following denervation. More recently Cardasis and Cooper ('75) have demonstrated that the total number of nuclei per muscle fiber (myonuclei plus satellite cell nuclei) does not change after denervation. Little is known about the effects of denervation upon nuclear populations in cat muscle, and the prolonged and irregular course of muscle fiber regeneration in the present experiment makes it difficult to determine whether or not there is an acceleration of the early stages in muscle fiber regeneration in pre-denervated cat muscle.

Mature muscle grafts always possess some morphologically abnormal elements. Scattered regenerating muscle fibers that are secondarily degenerating and small muscle fibers among normal appearing ones are found consistently in mature grafts. Muscle fibers with these characteristics may result because certain areas of the grafts do not become reinnervated. This conclusion is strengthened by the absence of heterogenous staining of the thin muscle fibers when stained for myofibrillar ATPase and succinic dehydrogenase activity (Maxwell et al., '77). In rats, the lack of differentiation of muscle fiber types is one of the chief abnormalities seen in muscle regenerating in the absence of nerves (Mong, '75; Carlson and Gutmann, '76).

Muscle spindles were not seen in several hundred complete cross sections of muscle grafts in this series. Yet occasional spindles have been seen in recent grafts over six months of age (unpublished observation). Hakelius et al. ('75) did not find muscle spindles in early grafts of cat peroneus muscles transplanted into tunnels in the intercostal region, but they also found spindles in older grafts. Muscle spindles are commonly seen in freely grafted rat muscles (Carlson and Gutmann, '75a).

Other abnormalities, such as increased 
amounts of connective tissue and bundles of irregularly oriented muscle fibers, could contribute to the deviation from normal of contractile properties of muscle grafts in the cat. No satisfactory explanation can be offered for the frequent persistence of central nuclei in muscle fibers in mature free grafts of cat muscle. Central nuclei are also commonly found in long term (4-month) muscle grafts in the rat (Carlson and Gutmann, '75a). In both cat and rat muscle grafts, central nuclei persist for a much longer period than they do during normal ontogenesis. The presence of central nuclei is a good marker for distinguishing grafted from normal muscle in microscopic preparations.

Despite some morphological and functional abnormalities, mature free grafts of both normal and pre-denervated EDL muscles in the cat possess a significant number of normal muscle fibers. Because of the similarity in size of cat muscle grafts to muscles that are currently being transplanted in humans, analysis of grafted muscles in the cat may provide a better understanding of the optimal preparation of human muscles for grafting as well as the course of events that results in a return of function to human muscle grafts.

\section{LITERATURE CITED}

Armed Forces Institute of Pathology 1960 Manual of Histologic and Special Staining Technics, Second ed. McGraw-Hill, N. Y., 67.

Bosova, N. N. 1962 Free autoplastic transplantation of whole muscies (In Russian). Byull. Exp. Biol. Med., 53(3); 88-92.

Cardasis, C. A., and G. W. Cooper 1975 A method for the chemical isolation of individual muscle fibers and its application to a study of the effect of denervation on the number of nuclei per muscle fiber. J. Exp. Zool., 191: 333346.

Carison, B. M. 1972 The Regeneration of Minced Muscles. Monographs in Devel. Biol. Vol 4. S. Karger AG, Basel, $128 \mathrm{pp}$.

1976 A quantitative study of muscle fiber survival and regeneration in normal, pre-denervated and Marcaine-treated free muscle grafts in the rat. Exp. Neurol., 52: 421.432 .

Carlson, B. M., and E. Gutmann 1975a Regeneration in free grafts of normal and denervated muscles in the rat: Morphology and histochemistry. Anat. Rec., 183: 47-62.

- 1975b Regeneration in grafts of normal and denervated rat muscles: Contractile properties. Pflugers Arch., 353: 215-225.

_- 1976 Contractile and histochemical properties of sliced muscle grafts regenerating in normal and denervated rat limbs. Exp. Neurol., 50: 319-329.

Hakelius, L. 1974 Transplantation of free autogenous muscle in the treatment of facial paralysis. Scand. J. Plast. Reconstr. Surg., 8: 220-230.

- 1975 . Free autogenous muscle transplantation in two cases of total anal incontinence. Acta Chir. Scand. 141: 69.75.

Hakelius, L., B. Nyström amd E. Stälberg 1975 Histochemical and neurophysiological studies of autotransplanted cat muscle. Scand. J. Plast. Reconstr. Surg., 9: 15-24.

Hsu, L. 1974 The role of nerves in the regeneration of minced skeletal muscle in adult anurans. Anat. Rec., 179: 119-136.

Maxwell, L. C., J. A. Faulkner, S. A. Mufti and A. M Turowski 1977 The free autografting of entire limb muscles in the cat: Histochemistry and biochemistry. J. Appl. Physiol., in press.

Mong, F. S. F. 1975 Histological and histochemical studies on the nervous influence on minced muscle regeneration of triceps surae of the rat. Ph.D. Thesis, University of Michigan, Ann Arbor.

Ontell, M. 1974 Muscle satellite cells: A validated technique for light microscopic identification and a quantitative study of changes in their population following denervation. Anat. Rec., 178: 211-228

Palmgren, A. 1960 Specific silver staining of nerve fibers. I. Technique for vertebrates. Acta Zool., 41: 239265.

Pearse, A. G. E. 1972 Histochemistry-Theoretical and Applied, Vol. 2. Third ed. Churchill Livingstone, Edinburgh, p. 1312.

Schiaffino, S., M. Sjöström, L. E. Thornell, B. Nyström and L. Hakelius 1975 The process of survival of denervated and freely autotransplanted skeletal muscle. Experimentia, 31: 1328-1330.

Snow, M. H. 1974 Origin of myoblasts during skeletal muscle regeneration. J. Cell. Biol., 63: 323a (Abstract).

Studitsky, A. N., and N. N. Bosova 1960 Development of atrophic muscular tissue in conditions of transplantation in place of mechanically damaged muscles. Arkh. Anat. Gist. Embriol., 39 (12): 18-32 (In Russian).

Thompson, N. 1971a Autogenous free grafts of skeletal muscle. Plastic Reconstr. Surg., 48: 11-27.

$\ldots \ldots 1971 \mathrm{~b}$ Investigation of autogenous skeletal muscle free grafts in the dog. Transplantation, 12: 353 . 363.

1974 A review of autogenous skeletal muscle grafts and their clinical applications. Clinics in Plastic Surgery, 1: 349-403.

Vyskočil, F., B. Carlson and E. Gutmann 1973 Changes in resting membrane potential and contractility of innervated and denervated skeletal muscle free grafts in the rat. Pfiugers Arch., 344: 181-186.

Yeasting, R. A. 1969 The effect of the nerve supply on the regeneration of minced skeletal muscle in the mouse Ph.D. Thesis, University of Louisville, Louisville.

Zhenevskaya, R. P. 1968 Transplantation of skeletal muscle in animals. Uspech. Sovrem. Biol., 65: 133-143 (In Russian).

Zhenevskaya, R. P., O. N. Rumyantseva, I. L. Novoselova and $E$. V. Proshlyakova 1965 Regenerative processes in a transplant of untreated muscle of young rats. Zhur. Obsch. Biol., 26: 569-576 (In Russian). 
PLATES 
PLATE 1

\section{EXPLANATION OF FIGURES}

1 Four-day graft of pre-denervated EDL muscle. The fascicular architecture of the original muscle remains. At the top of the section (arrow), inflammatory cells are beginning to occupy the peripheral perimysial connective tissue. There is little sarcolysis of old muscle and no histological evidence of regeneration. $\mathrm{H} \& \mathrm{E}$. $\times 37.1$.

2 Eight-day graft of pre-denervated EDL muscle, showing the earliest time at which the three major zones of histological activity are present. At the periphery $(P)$ are intact original muscle fibers that have apparently survived the grafting procedure. In the center $(\mathrm{C})$, still occupying much of the graft, are necrotic muscle fibers that have undergone degenerative changes, but not extensive loss of sarcoplasm. In addition to the lighter than normal staining, the degenerating muscle fibers show characteristic transverse fissures (arrows). Between these two areas is the intermediate zone in which the original muscle fibers have undergone sarcolysis and are replaced by regenerating muscle fibers. H \& E. $\times 112.0$.

3 Twenty eight-day graft of pre-denervated EDL muscle. The central zone (C) of necrotic original muscle fibers has shrunk considerably. This area is surrounded by regenerating muscle fibers that are already arranged into fasciculi. H \& E. $\times \mathbf{4 4 . 1}$.

4 Higher power section through the same 28-day graft as that illustrated in figure 3 . This section shows the radial gradient of more mature regenerating (and possibly some surviving) muscle fibers at the periphery (top) and less mature regenerating muscle fibers toward the center of the graft (arrows). At the very bottom of this figure are a few necrotic original muscle fibers located in the central zone (C). H \& E. $\times \quad 77.0$. 

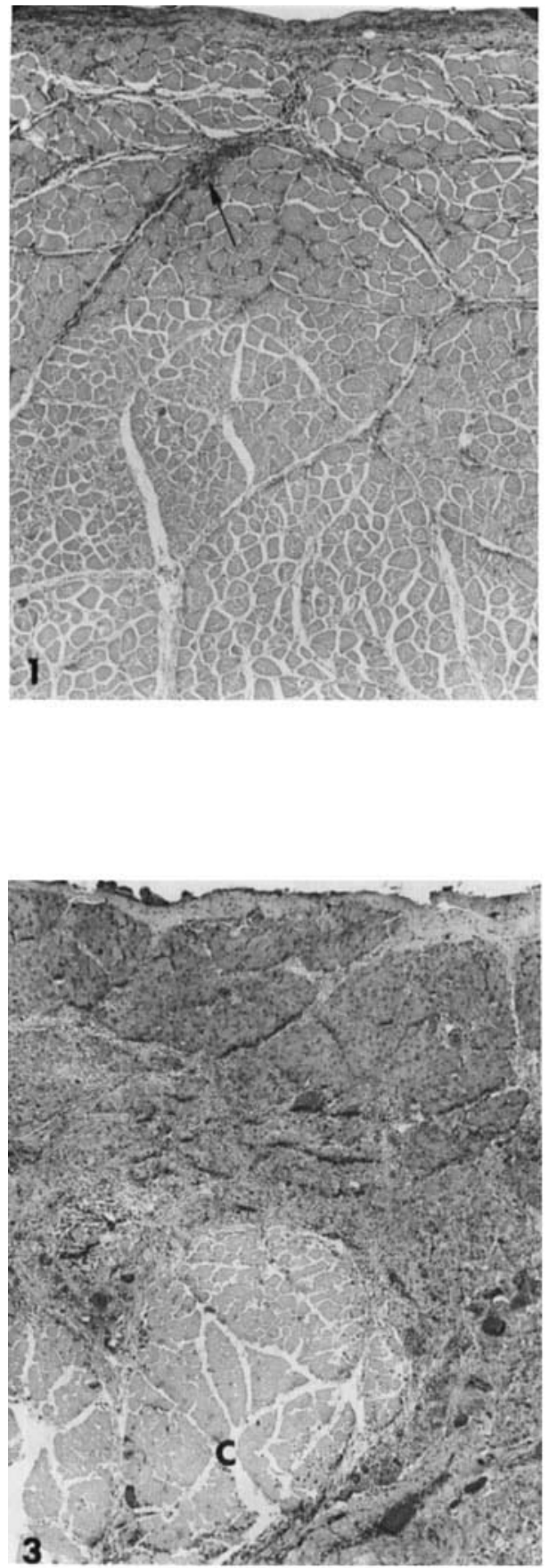
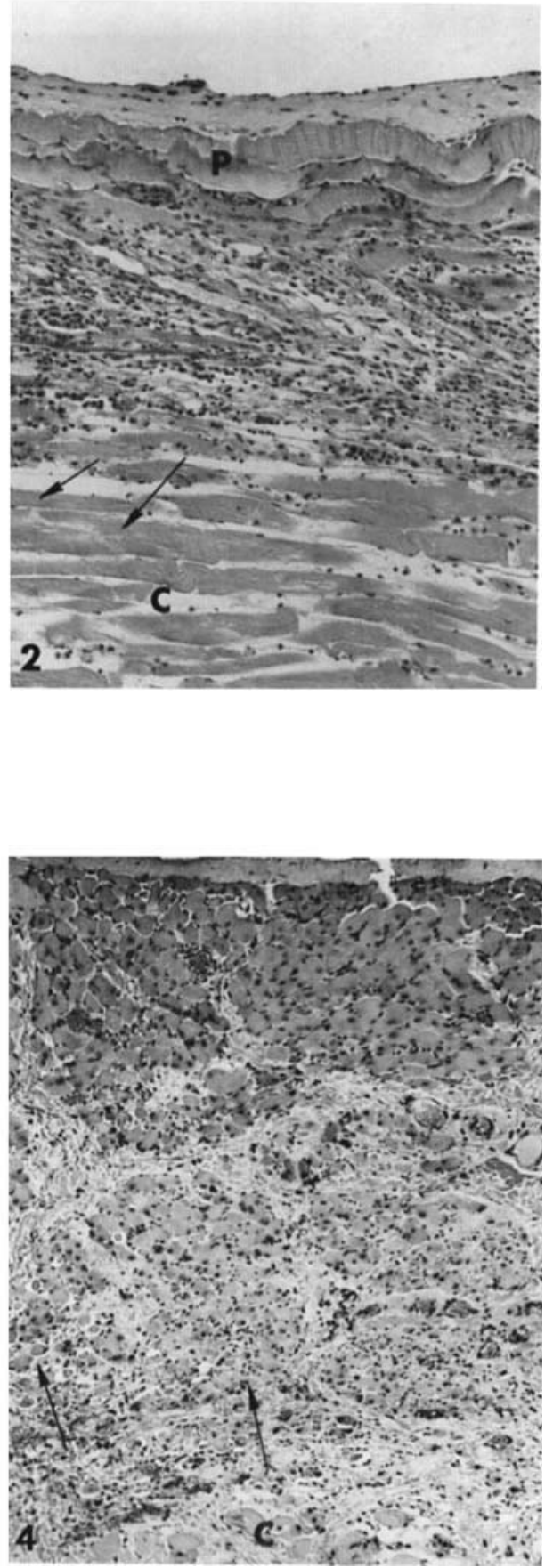


\section{PLATE 2}

EXPLANATION OF FIGURES

5 Forty-one-day graft of pre-denervated EDL muscle showing the gradient of maturation of muscle fibers from the periphery (top) to the center (bottom) of the graft. The old muscle fibers of the central zone have been resorbed, and the center of the graft is occupied by loose connective tissue, macrophages and some immature muscle fibers. A dense casing of connective tissue (arrow) surrounds the graft. H \& E. $\times 36.4$

6 Six-month graft of pre-denervated EDL muscle. Mature muscle fibers and connective tissue fill the entire graft. H \& E. $\times 9.9$.

7 Six-month graft of pre-denervated EDL muscle showing large numbers of very thin muscle fibers (arrows) scattered among fascicles of muscle fibers of normal diameter. H \& E. $\times 84.0$

8 Longitudinal section through the same graft as that shown in figure 7 . H \& E. $\times 91.0$. 
FREE MUSCLE GRAFTS IN THE CAT

PLATE 2

S. Mufti, B. Carlson, L. Maxwell and J. Faulkner
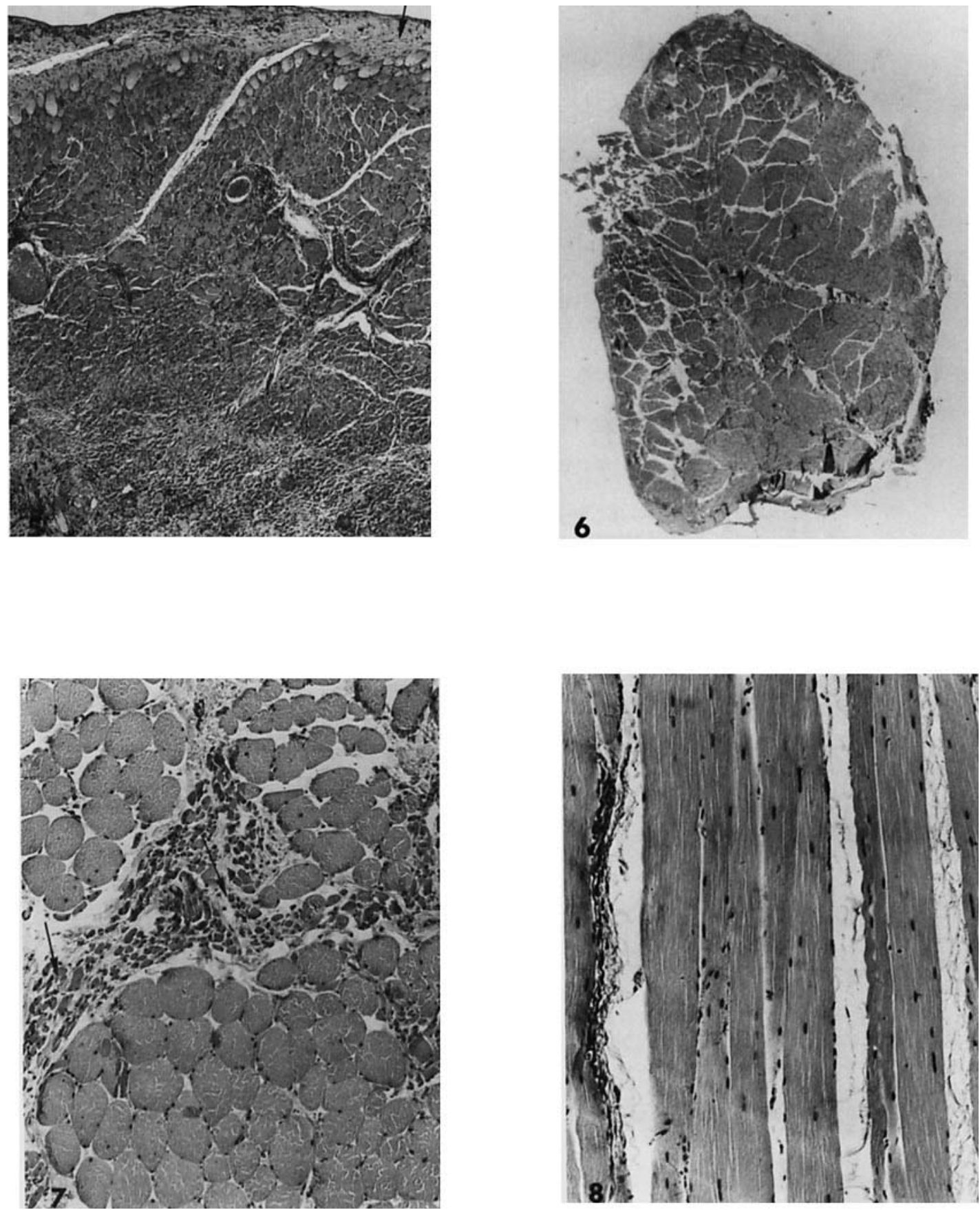
PLATE 3

EXPLANATION OF FIGURES

9 Eight-day graft of normal EDL muscle, showing a large central area (C) of palestaining original muscle fibers. At the periphery is a thin zone of degeneration of old muscle fibers and early regeneration of new. H \& E. $\times 42.1$

10 High power view throurh the periphery of the graft in figure 9 , showing a thin rim of apparently surviving muscle fibers (S). Immediately beneath them are degenerating muscle fibers (D), which are penetrated to a varying extent by macrophages engaged in sarcoplasmic removal. Other muscle fibers (C) of the central area have not yet been invaded by macrophages. H \& $\mathrm{E} . \times 210.0$.

11 Twenty six-day graft of pre-denervated EDL showing some of the degenerative changes seen in regenerating muscle fibers. 1 , nuclear pycnosis, 2 , increased intensity of cytoplasmic staining; 3 , stacking of nuclei and/or nuclear fragments. $\mathrm{H} \& \mathrm{E}$. $\times 175.0$. 
FREE MUSCLE GRAFTS IN THE CAT

PLATE 3
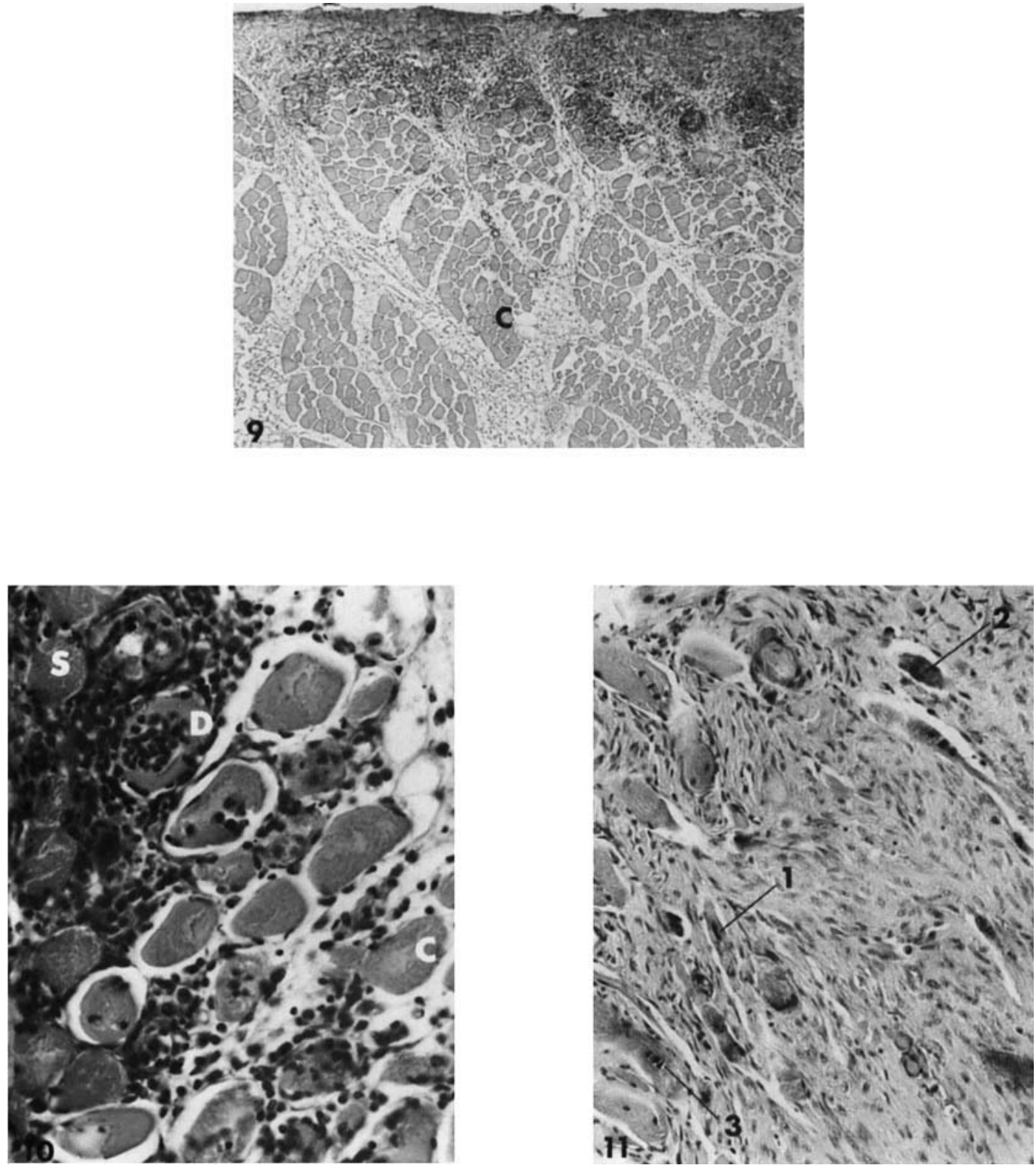